\title{
CATHARE \\ Description, exemple de calcul d'un accident complet de réacteur PWR
}

\author{
The CATHARE system : \\ Description and example of a completely simulated accident in a PWR
}

G. Houdayer, J.M. Miraucourt*

EDF, Direction de l'Equipement

\section{Le code CATHARE}

\subsection{Objectif}

CATHARE (Code avancé de Thermo-Hydraulique pour Accidents des Réacteurs à Eau) est un programme de simulation des accidents de perte de réfrigérant primaire (APRP) pour les réacteurs nucléaires à eau pressurisée.

Développé conjointement par EDF, CEA et Framatome dans le cadre d'une équipe mixte d'une vingtaine d'ingénieurs implantée au Centre d'études nucléaires de Grenoble, CATHARE a pour objectif le calcul "réaliste " des APRP, afin d'estimer les marges de sécurité adoptées jusqu'à présent après l'analyse de ces accidents à l'aide des codes américains dits de première génération. Parallèlement au développement du code, un important programme expérimental analytique, mené sur une période de dix ans (1974-1984), permet la qualification des modèles physiques de CATHARE. Le contrôle des capacités du code à effectuer la transposition de ces essais analytiques (qui concernent des phénomènes ou des composants particuliers) à des circuits complexes se fait par la simulation d'essais globaux (tels LOFT aux USA ou BETHSY qui sera réalisé à Grenoble).

Sans compter la réalisation des essais ni les supports antérieurs ou parallèles reçus par l'équipe mixte, la première version de CATHARE, aujourd'hui opérationnelle, a nécessité pendant trois ans un travail à la fois théorique (mise en forme des modèles), expérimental (dépouillement des essais), numérique (mise au point d'analyses numériques sûres) et informatique (acquisition et gestion des données, traitement des résultats).

CATHARE constitue également la base de réalisation d'un simulateur d'accidents capable de représenter en temps réel le déroulement de transitoires complexes. Ce développement est réalisé par une équipe mixte EDF CEA - Thomson également implantée à Grenoble.

\subsection{Hypothèses de départ}

La simulation du réacteur est limitée au circuit primaire. Seuls sont pris en compte les phénomènes qui influent directement et fortement sur le comportement thermohydraulique de l'écoulement diphasique (eau + vapeur) :

- production, déstockage et transferts d'énergie : neutronique, réaction chimique Zircalloy + eau et échanges $\mathrm{UO}_{2}$-gaine-fluide dans le cœur; échanges primaire secondaire dans les générateurs de vapeur; échanges parois - fluide;

- déformation et rupture des gaines des crayons combustibles;

- vitesse des pompes.

Les phénomènes extérieurs au circuit primaire (circuit secondaire, systèmes de sécurité, enceinte) ne sont pas calculés, mais apparaissent sous forme de conditions aux limites simples pour le calcul du circuit primaire.

Enfin CATHARE ne représente pas le comportement des cœurs fortement dégradés et n'effectue aucun calcul d'efforts sur les composants du circuit.

\subsection{Modèles}

L'écoulement de l'émulsion diphasique joue le rôle central dans le déroulement des APRP. Une attention toute particulière a été apportée à la modélisation de ces écoulements. Le modèle retenu (axial à 6 équations, voir exposé réf. [1]), permet de représenter les écoulements très divers présents dans le circuit en situations accidentelles,

(*) Equipe mixte EDF/CEA/Framatome, CEN, Grenoble

LA HOUILLE BLANCHE/No 3/4-1984 
que ces écoulements soient émulsionnés ou à phases séparées, verticaux ou horizontaux, stratifiés ou non, cocourant ou contre-courant, que les phases soient à l'équilibre thermodynamique ou en état métastable.

Le comportement des volumes du circuit est représenté par un modèle équivalent dans lequel les équations de moment ont été simplifiées (hypothèse de vitesse faible à l'intérieur des volumes) (voir exposé réf. [2]).

Les tés, permettant les piquages et les raccordements d'une canalisation sur une autre, ont un modèle proche du modèle d'écoulement. Les équations d'impulsion, écrites sur chacune des trois branches, sont ajoutées vectoriellement.

La modélisation des pompes est faite par une transposition en diphasique des courbes hauteur et couple établies en monophasique. Ceci permet le calcul de l'interaction pompes-fluide et la vitesse de rotation dans les différents quadrants (débits et/ou vitesse de rotation positifs ou nuls).

Dans les canalisations, un calcul de conduction dans la paroi permet de représenter le déstockage de l'énergie dans le métal (phénomène important en cas de transitoire lent).

Dans le cơur, à la modélisation axiale à 6 équations de l'écoulement, est associée la modélisation du comportement thermomécanique du combustible : à chaque cote, on effectue un calcul de conduction radiale dans $\mathrm{I}^{\prime} \mathrm{UO}_{2}$, le jeu $\mathrm{UO}_{2}$-gaine (éventuellement oxydée en surface). En cas de renoyage du cœur, un calcul de conduction bi-dimensionnelle (axial et radial) permet de représenter les gradients de températures et les déstockages d'énergie très importants au voisinage du front de trempe. Les déformations de la gaine (dilatation, domaine élastique, fluage) sont représentées, pouvant aller jusqu'à la rupture.

La réaction exothermique $\mathrm{Zr}+\mathrm{O}_{2}$ qui peut se déclencher vers $700^{\circ} \mathrm{C}$ et participer de façon importante au bilan énergétique et à la dégradation du coœur, est modélisée.

La puissance nucléaire est calculée par un modèle de cinétique neutronique ponctuelle prenant en compte les antiréactivités dues à l'effet Doppler, à la densité du modérateur et à la descente des barres de contrôle.

\subsection{Aspects numériques}

L'ensemble des équations obtenues est résolu par une méthode aux différences finies (discrétisation en espace et en temps).

La discrétisation en espace est dite «à mailles décalées " (les points où sont calculées les pressions, enthalpies et taux de vide sont intercalés avec les points où sont calculées les vitesses). La discrétisation adoptée est convergente et monotone.

L'ordre de grandeur du nombre de points utilisés pour représenter un circuit réacteur est de l'ordre de quelques centaines.

La discrétisation en temps est implicite. Cette méthode permet l'utilisation de grands pas de temps lorsque le transitoire est relativement lent.

Après discrétisation, on obtient un système d'équations algébriques non linéaire, permettant le calcul de toutes les inconnues au temps $t$, connaissant l'état du circuit au temps précédent $t-\Delta t$. En transitoire, le système est résolu par la méthode itérative de Newton. A chaque itération, la linéarisation du système permet une structuration modulaire du code; chaque module, à partir du jacobien des équations qui le concernent, déduit les relations linéaires qui lient, à l'itération en cours, les variables à ses jonctions. L'assemblage de tous les modules se fait ensuite par la réunion des relations fournies par chaque module, le système obtenu ne portant que sur les variables aux jonctions. Enfin, à partir de ces valeurs aux jonctions, chaque module calcule ses inconnues internes.

Cette méthode permet de concilier les aspects numériques essentiels du code : non linéaire, implicite, modulaire.

L'initialisation à l'instant $t=0$ fait l'objet d'un calcul particulier. Effectué avec les mêmes modèles qu'en transitoire, ce calcul suppose que le circuit est en régime permanent stable. Les algorithmes mis au point pour l'équilibrage du circuit (répartitions de débit, pertes de charges) permettent la représentation de topologies complexes de façon très économique en temps calcul.

\subsection{Aspects informatiques}

L'acquisition des données (en format libre) et la description de la séquence accidentelle (langage externe permettant les commandes et interventions depuis le jeu de données) font de CATHARE un code souple et simple d'emploi.

L'utilisateur dispose de commandes de description, de contrôle, d'exécution et d'exploitation des résultats, qui le rendent maitre (et responsable) de l'assemblage des différentes pièces modulaires du code.

CATHARE représente environ 300 sous-programmes (50 000 cartes) écrits en langage FORTRAN. Il est actuellement implanté sur le calculateur CRAY.1 des réseaux CISI et EDF.

\subsection{Etat d'avancement}

CATHARE est aujourd'hui opérationnel. Une phase de mise au point importante, sur des calculs réacteurs du type petite brèche, vient de s'achever.

La vérification sur l'ensemble du programme expérimental analytique est en cours, afin de confirmer la capacité du code à reconstituer les essais physiques qui ont servi de base à sa construction.

La simulation d'essais globaux (LOFT) vient de commencer.

Parallèlement, la poursuite de la simulation d'accidents grosse brèche permet la mise au point du code dans les phases de remplissage et de renoyage.

Le code, ainsi que sa documentation détaillée (dont la rédaction est en cours) sera disponible dès la fin de cette année 1983 dans sa version CATHARE 1.

Des développements numériques et physiques se poursuivent dans l'optique de la réalisation d'une future version CATHARE 2 du code. 
2. Exemple de calcul réacteur : APRP petite brèche (6 pouces)

\subsection{Représentation du circuit}

L'accident calculé est la rupture du piquage d'une tuyauterie de diamètre 15 centimètres ( 6 pouces) sur l'une des trois branches froides du réacteur.

La figure I présente la modélisation du circuit primaire retenue. Elle comprend 13 tuyaux, 11 volumes, 2 pompes et 2 tés.

Le pressuriseur est situé sur la boucle rompue. Les deux autres boucles, supposées avoir un comportement identique, ne sont représentées que par l'une d'entre elles.

Le cœur est modélisé par un canal simulant le comportement moyen des 41448 crayons combustibles du réacteur.

La brèche, de type boutonnière, est représentée par un piquage té - tuyau au milieu de la branche froide.

\subsection{Régime permanent}

Au temps $t=0$, l'état du réacteur, supposé être en fonctionnement normal, doit être conforme aux spécifications fournies par le constructeur et prendre en compte les diverses hypothèses de sûreté propres à ce type d'accident. En particulier, doivent être respectés :

- la puissance du cœur : $102 \%$ de la puissance nominale, soit $2830 \mathrm{MW}$;

- la pression au dôme supérieur du pressuriseur: la valeur normale majorée de 2 bars, soit 157,2 bars;

- les débits de chacune des boucles : $21960 \mathrm{~m}^{3} /$ heure;

- le débit de by-pass du cœur (eau traversant le cœur mais non en contact avec des crayons combustibles): $4,5 \%$ du débit total;

- le débit sous le couvercle : $2 \%$ du débit total, fluide froid;

- la vitesse de rotation des pompes : 1485 tours/minute.

La figure 2 montre que le régime permanent obtenu par CATHARE répond parfaitement à ces exigences. On constate aussi :

- un bon équilibrage des boucles en débit : $0,1 \%$ d'écart de débit entre elles:

_ un bon équilibrage thermique des boucles : $948,6 \mathrm{MW}$ extraits à chaque générateur de vapeur.

\subsection{Interventions extérieures (accident et automatis- mes)}

Au temps $t=0$, la brèche est ouverte en 3 millisecondes. Les différents automatismes à simuler sont :

- l'arrêt d'urgence (sur signal basse pression : 129 bars au pressuriseur) : il se traduit par la chute des barres de contrôle (qui va provoquer la décroissance de la puissance produite au cœur) et l'isolement des générateurs de vapeur (coupure au secondaire de l'eau alimentaire et de la vapeur extraite). On suppose également la perte de l'alimentation électrique des pompes primaires:

- les injections de secours: sont simulés les divers systèmes de sauvegarde destinés à assurer le refroidissement du cœur :

- injection de secours haute pression : déclenchement trente secondes après le signal de très basse pression (117 bars) au pressuriseur,

- déclenchement des accumulateurs : des réservoirs d'eau froide pressurisés à l'azote débitent dans le circuit primaire lorsque la pression y devient inférieure à 41 bars,

- déclenchement de l'alimentation de secours des générateurs de vapeur: 60 secondes après l'arrêt d'urgence, un débit d'eau alimentaire ( $4 \%$ du débit nominal) est rétabli au secondaire.

D'autre part, des soupapes tarées à 74 bars limitent la montée en pression du secondaire des générateurs de vapeur.

La figure 3 résume ces interventions.

\subsection{Déroulement de l'accident}

Quatre grandes phases peuvent être distinguées:

- de 0 à 80 secondes environ: L'ouverture de la brèche provoque la décompression rapide du circuit jusqu'à 75 bars. Cette décompression entraîne l'apparition de vapeur dans les parties chaudes du circuit (cœur, plénum supérieur, branches chaudes et générateurs de vapeur). Le pressuriseur ( 16 tonnes d'eau à $t=0 \mathrm{~s}$ ) se vide de son eau par vidange dans le circuit primaire et par vaporisation (pressuriseur complètement en vapeur dès $t=22 \mathrm{~s}$ ).

La puissance produite au cœur décroît fortement après la chute des barres et tend rapidement vers son niveau résiduel ( $2 \%$ de la puissance nominale).

L'isolement des générateurs de vapeur provoque la montée en pression du secondaire, jusqu'à l'ouverture des soupapes à 74 bars.

- de 80 à 176 secondes : cette phase est caractérisée par un palier de pression primaire à 75 bars. A cette pression, l'écart de température au générateur de vapeur entre primaire et secondaire (à saturation et à pression constante : 74,4 bars) est suffisant pour permettre l'extraction de la puissance résiduelle produite au cœur.

La forte diminution de la vitesse de rotation des pompes provoque un ralentissement général du fluide dans le circuit. Les phénomènes gravitaires deviennent prépondérants: l'eau "se tasse " dans les parties basses du circuit, avec apparition de stratification dans les volumes et les tuyaux.

La diminution du débit dans le cœur y provoque une forte vaporisation et l'apparition d'une zone de découvrement à sa partie supérieure. L'énergie produite dans la partie découverte des crayons est très mal évacuée par la vapeur sèche: la température des gaines monte.

La vapeur surchauffée produite au cœur part dans les branches chaudes puis est partiellement recondensée dans la partie montante des générateurs de vapeur le long desquels ruisselle un film liquide (fonctionnement en caloduc).

L'eau, retombée des parties hautes du circuit (couvercle, générateurs de vapeur) et tassée au fond du circuit, forme un bouchon dans les branches intermédiaires en $\mathrm{U}$. La brèche reste en phase liquide.

La dégradation des échanges au primaire des générateurs de vapeur (presque en vapeur sèche) provoque une 


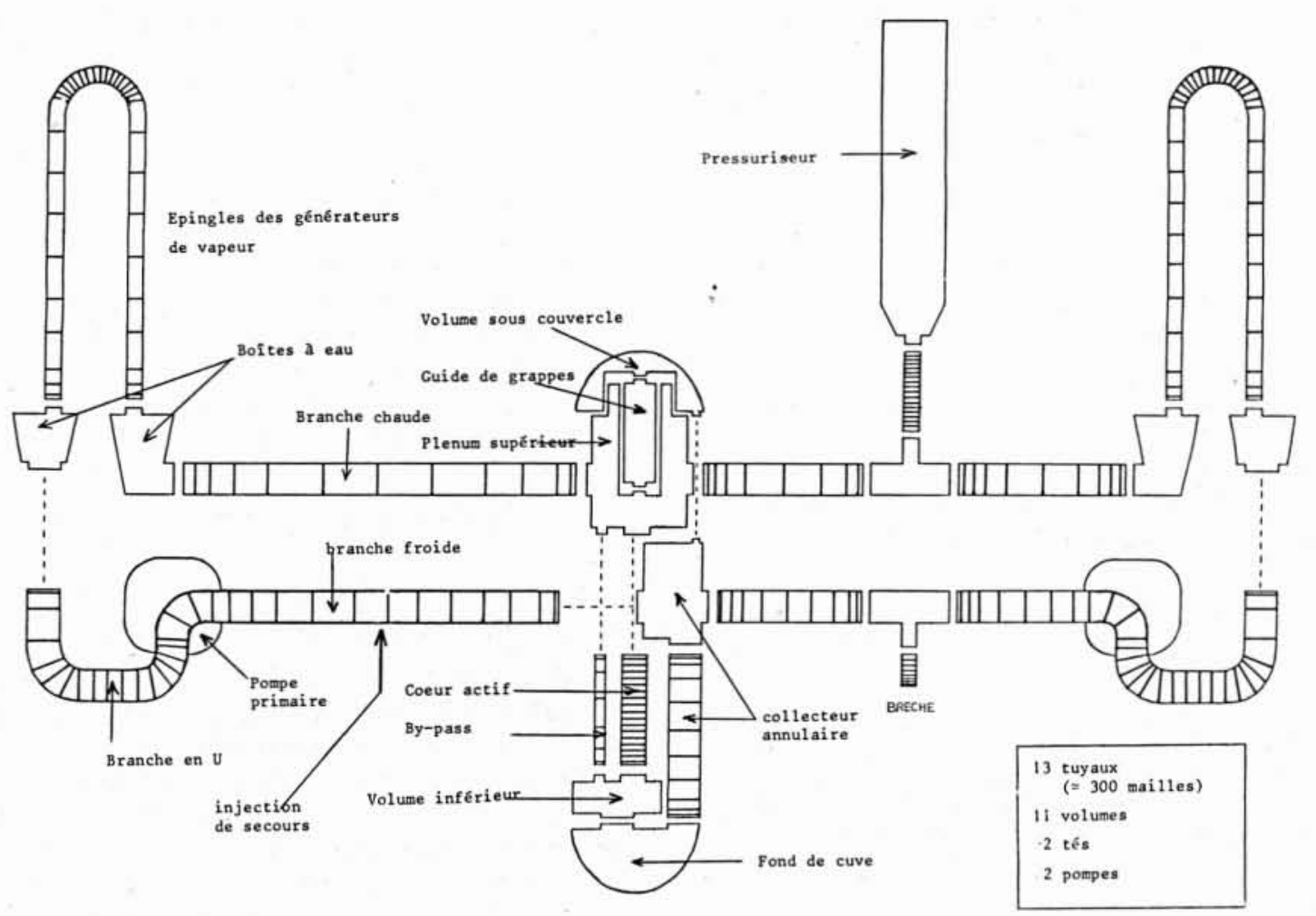

Figure 1. - Modélisation CATHARE du circuit primaire (réacteur PWR 900 MW type CP1)

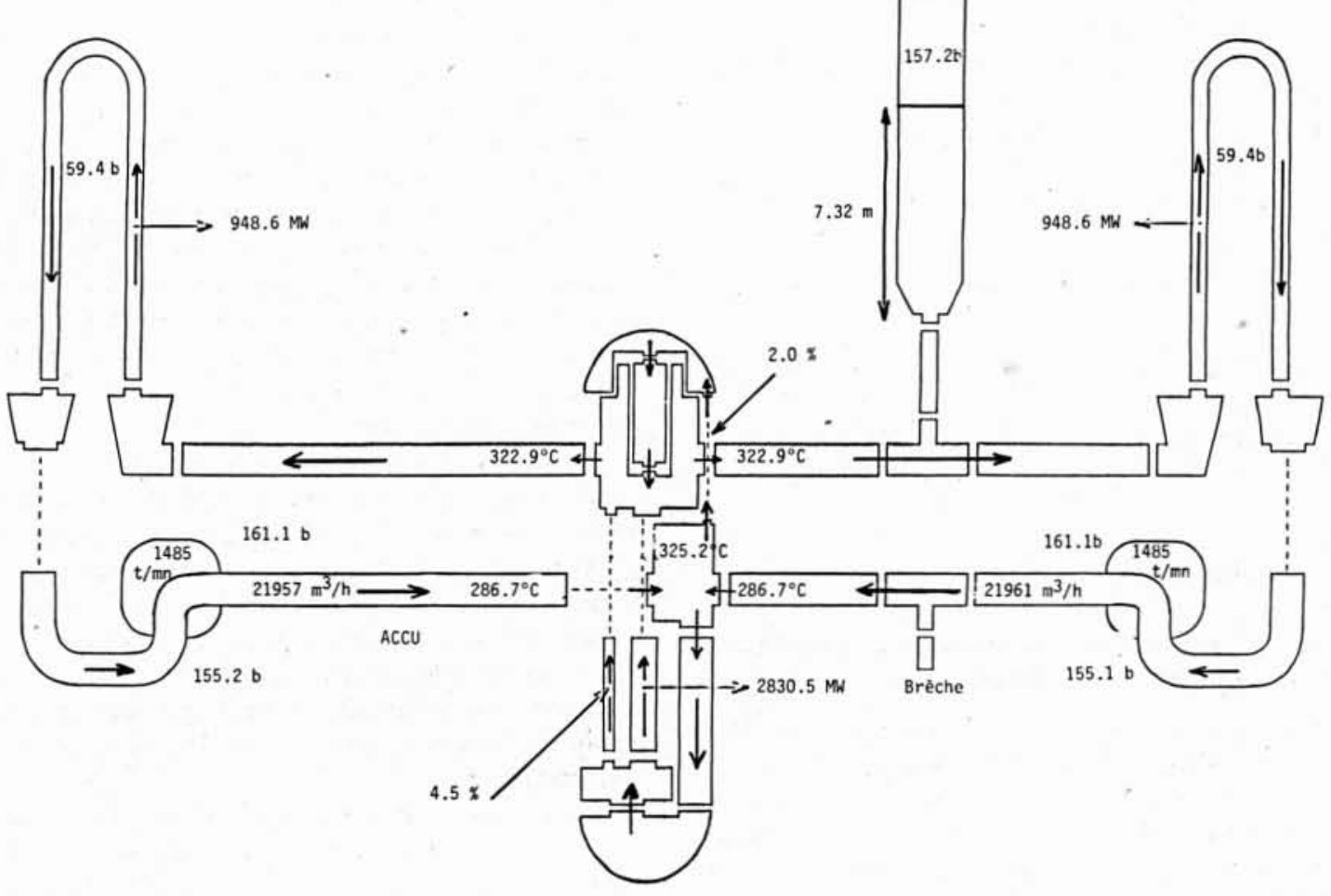

Figure 2. - Régime permanent 


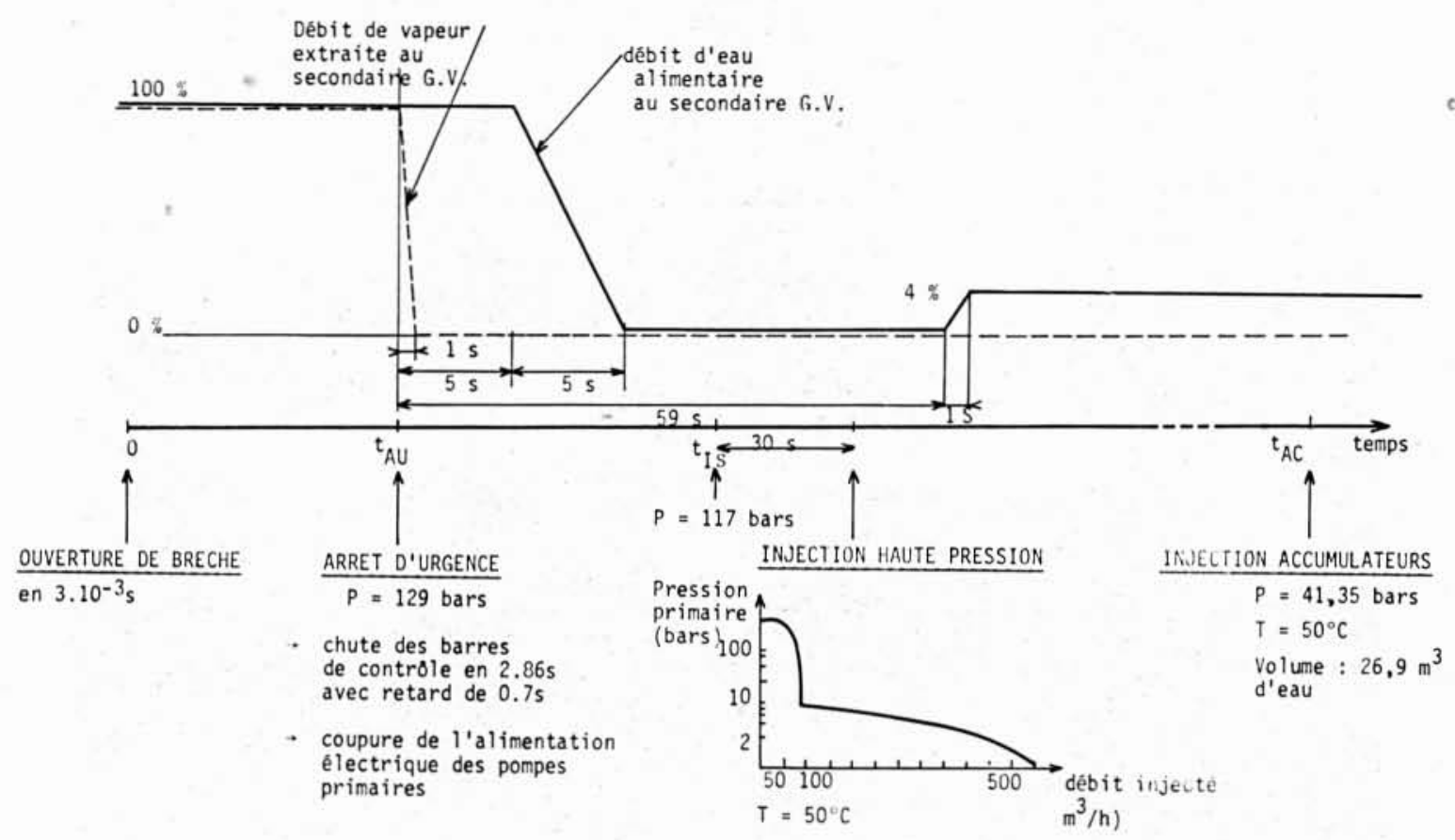

Figure 3. - Description des interventions.

Signal d'arrêt d'urgence

Signal ISHP

Ouverture soupapes G.V.

intact

Fin de vidange du pressuriseur Injection ISHP

Fermeture soupapes G.V.

rompu

intact

Découvrement du cœur (haut)

Température maximum de gaine

Début chasse bouchon d'eau

Injection accumulateurs

intact

intact

\begin{tabular}{|r|} 
CATHARE \\
$6.6 \mathrm{~s}$ \\
$8.5 \mathrm{~s}$ \\
$13 . \mathrm{s}$ \\
$18 . \mathrm{s}$ \\
$22 . \mathrm{s}$ \\
$38.5 \mathrm{~s}$ \\
$140 . \mathrm{s}$ \\
$110 . \mathrm{s}$ \\
$140 .-200 . \mathrm{s}$ \\
$197 . \mathrm{s}$ \\
$170 . \mathrm{s}$ \\
$190 . \mathrm{s}$ \\
$395 . \mathrm{s}$ \\
\hline
\end{tabular}

Figure 4. - Chronologie

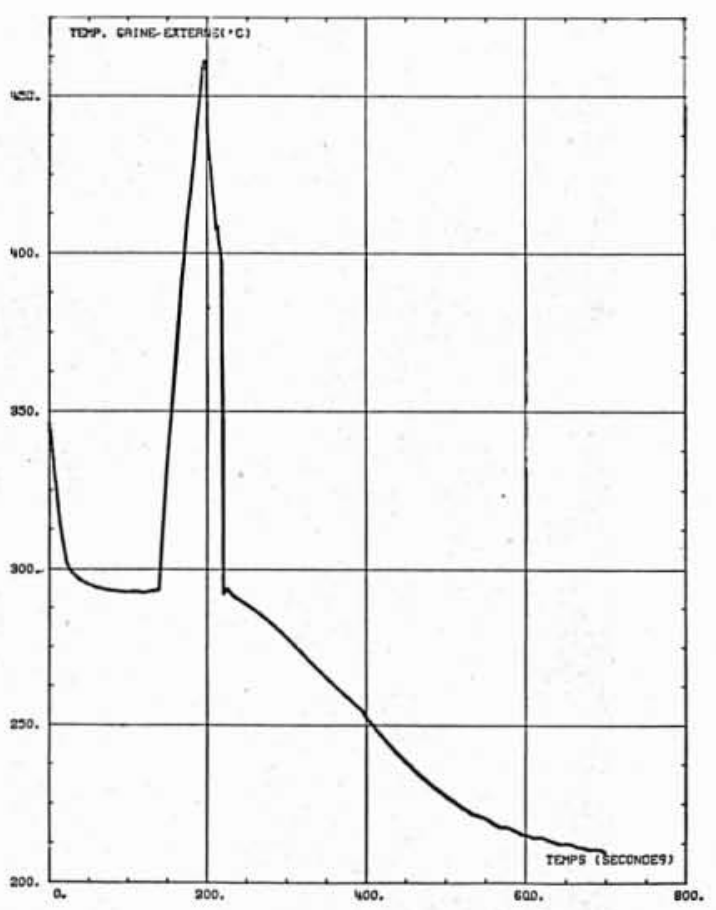

Figure 5. - Evolution de la température de la gaine externe en fonction du temps au point $z=3,200 \mathrm{~m}$ de lélément COEURMOY. 


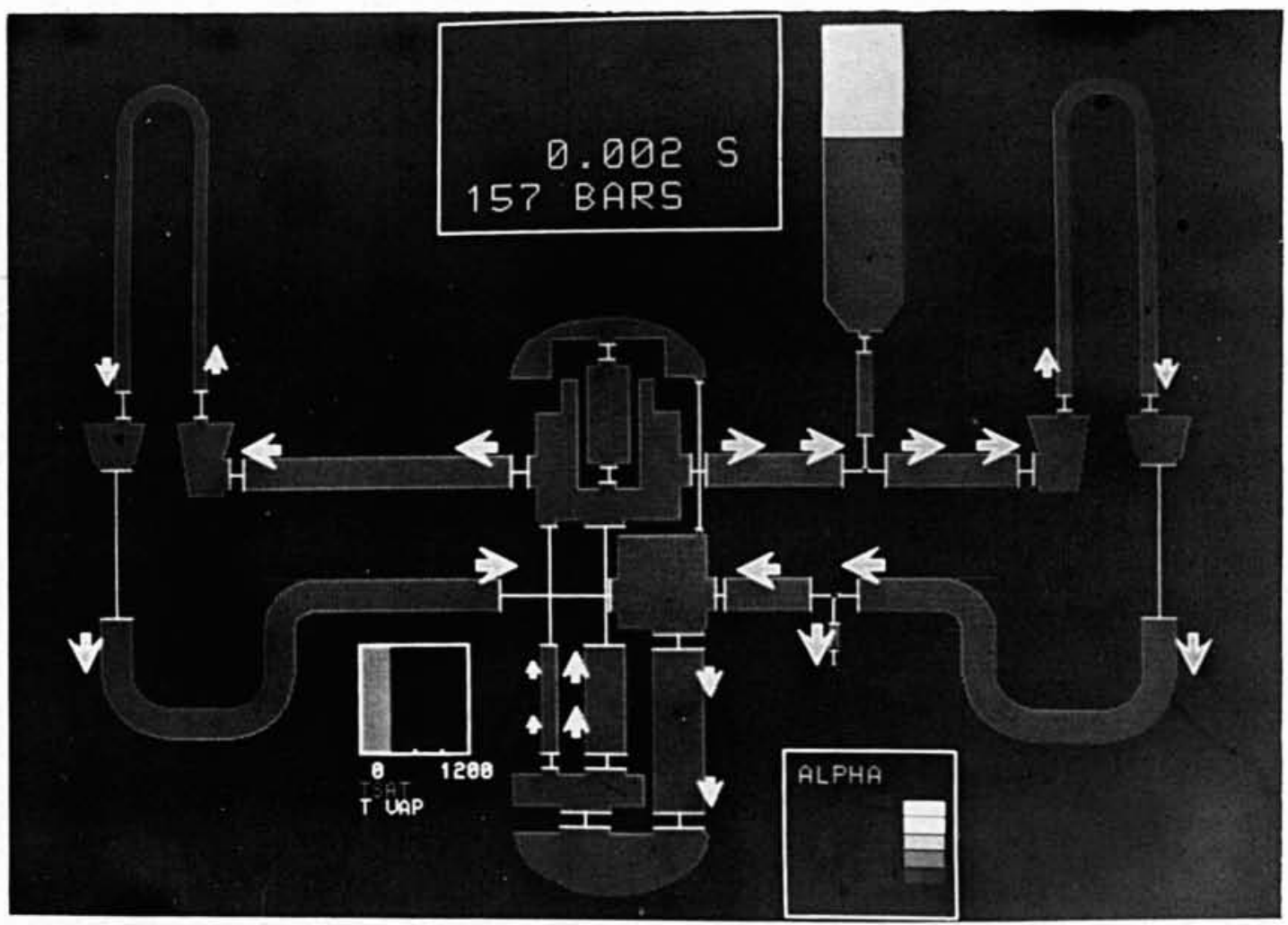

Figure 6

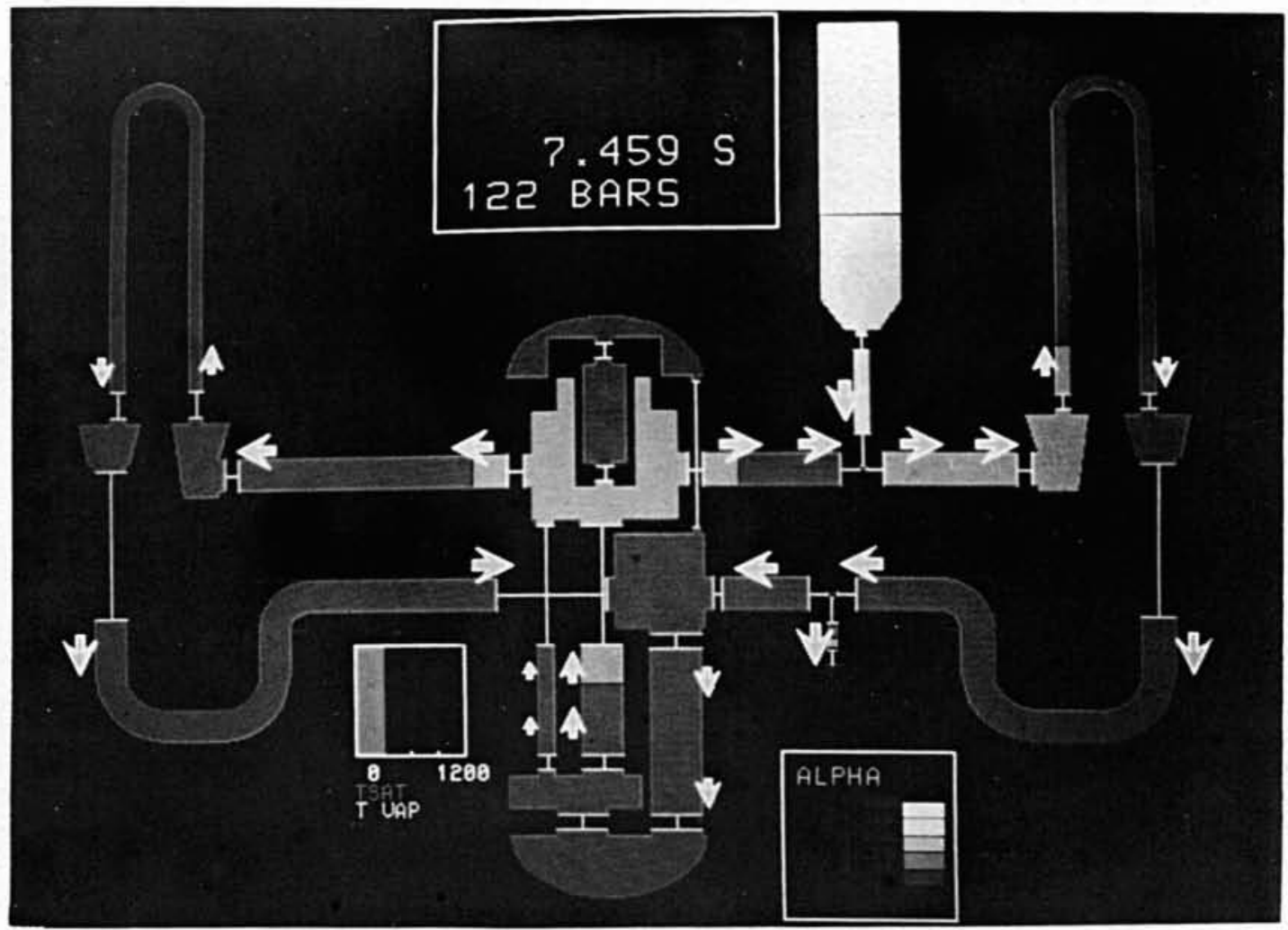

Figure 7 


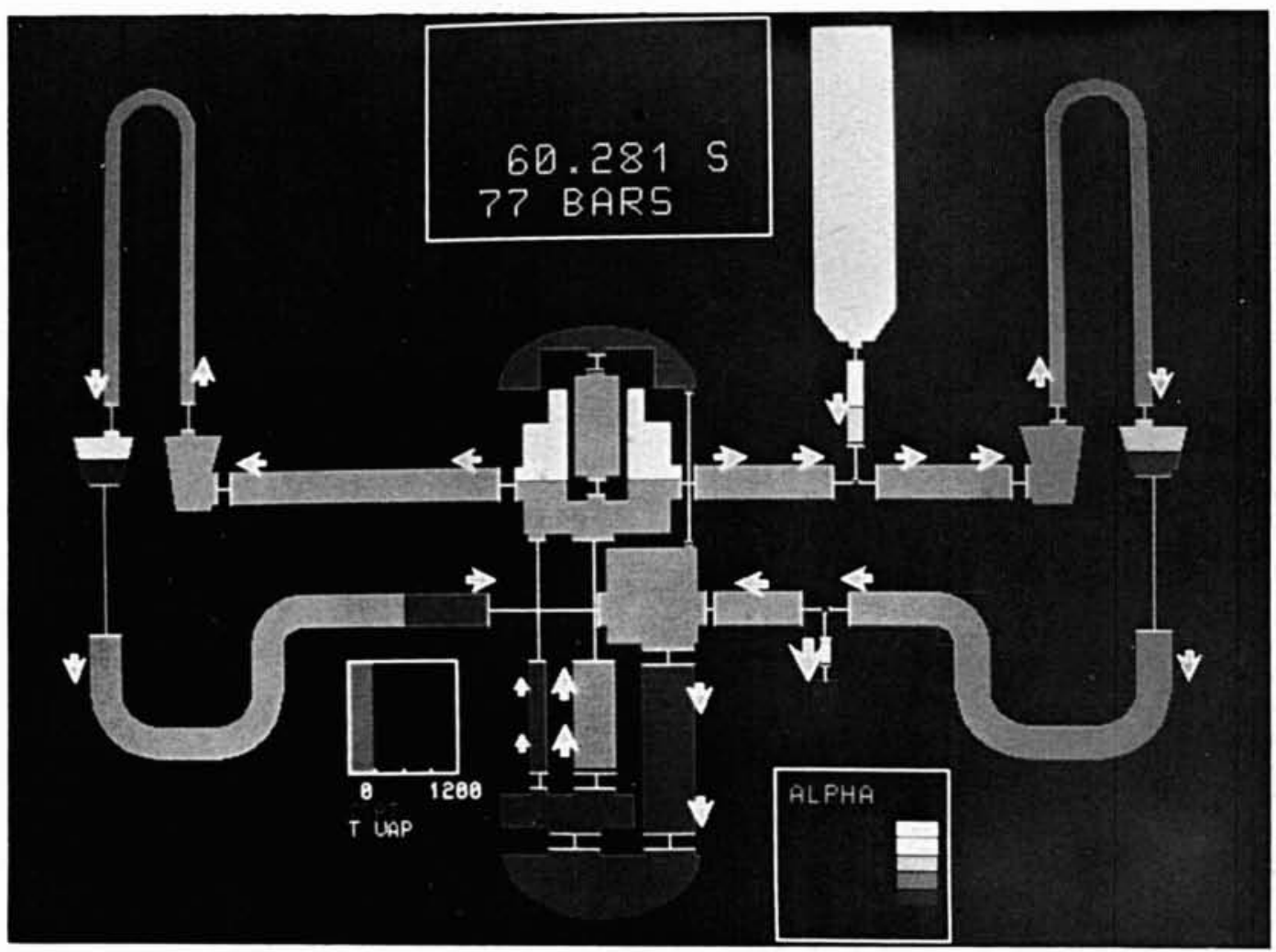

Figure 8

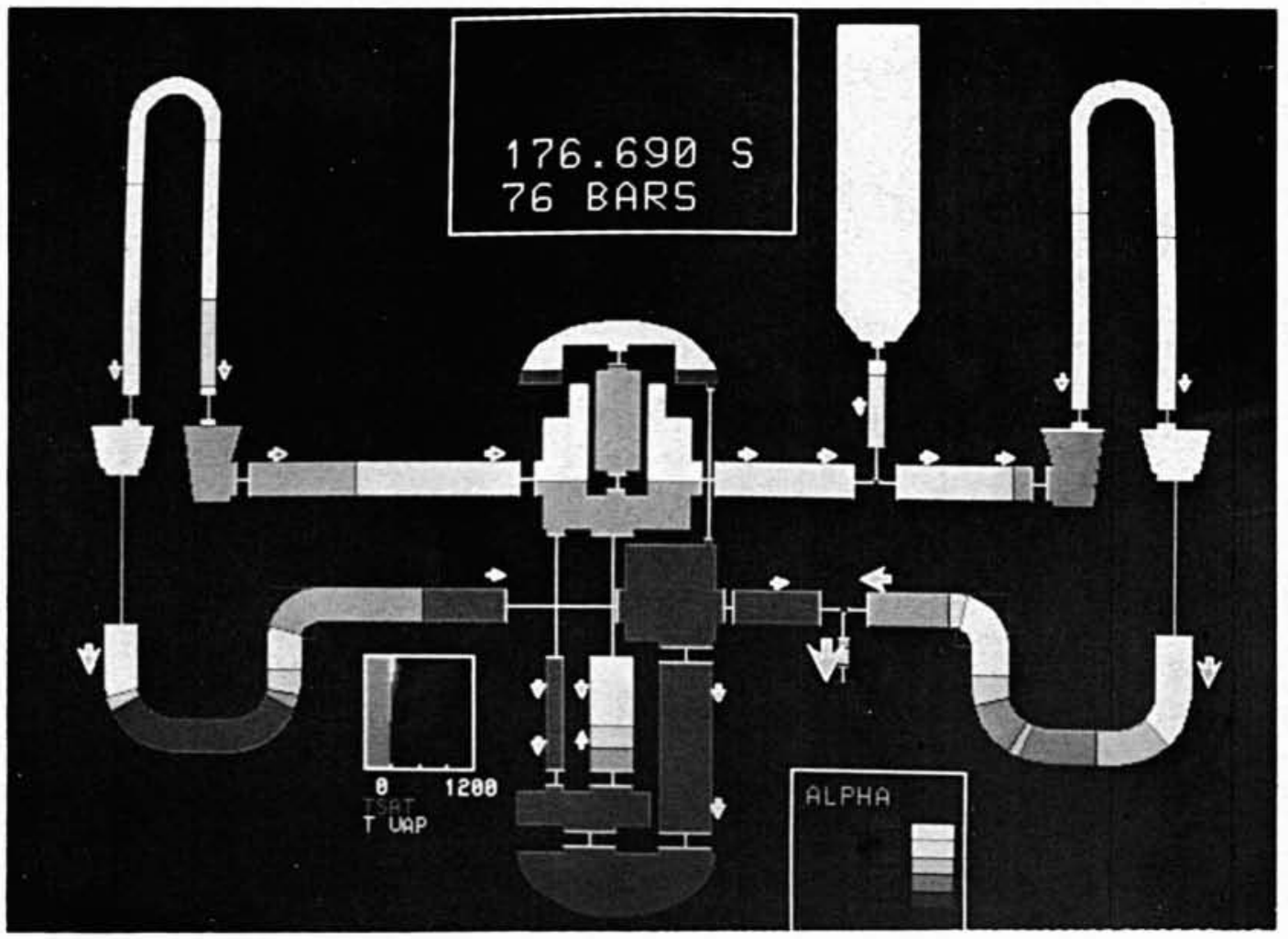

Figure 9 


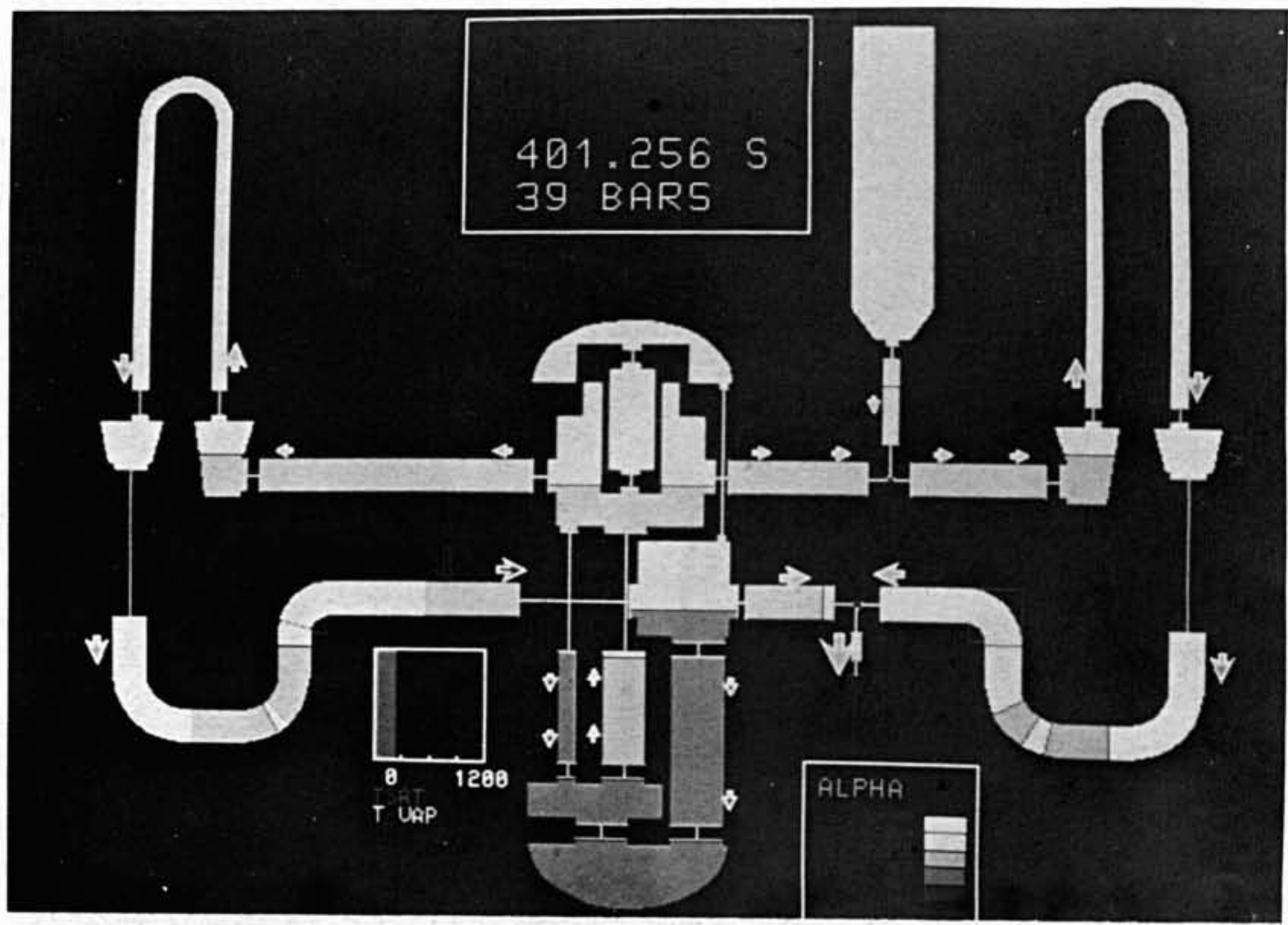

Figure 10

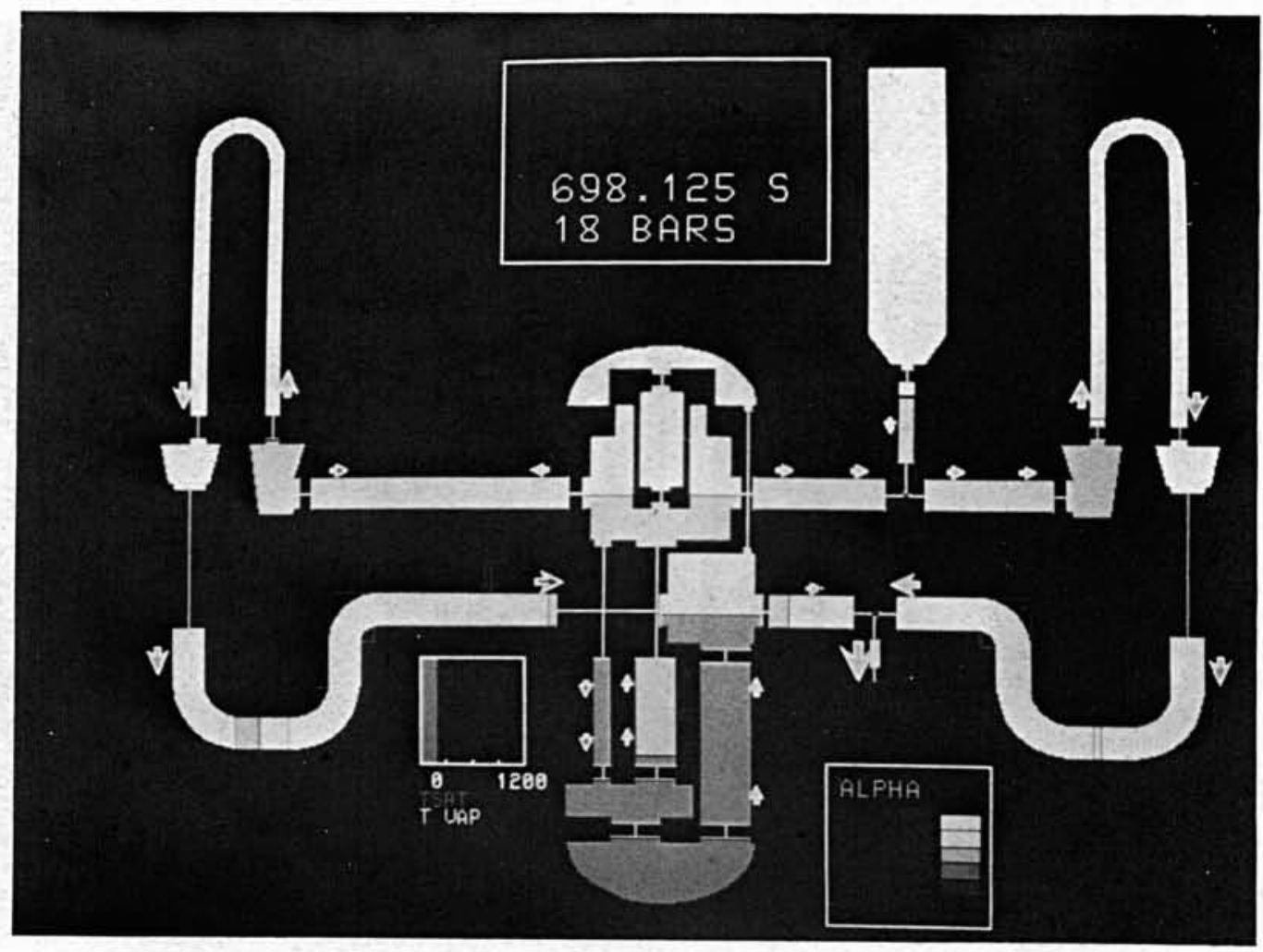

Figure 11 
légère remontée de la pression primaire afin d'assurer l'extraction de la puissance produite au cœur. Cette surpression pousse les bouchons d'eau dans les branches en $U$ et finit par les chasser.

- de 176 à 400 secondes: la chasse des bouchons d'eau a lieu à 176 secondes du côté rompu et à 195 secondes du côté intact. Dès le passage de la brèche en phase vapeur, la pression primaire chute rapidement ( 41 bars à 400 secondes).

La disparition des bouchons d'eau entraîne une brusque augmentation des vitesses dans le circuit et provoque le recouvrement du cœur par l'émulsion diphasique. Les échanges fluide-combustible sont à nouveau bons et la température des gaines chute.

- de 400 à 700 secondes: la pression de déclenchement des accumulateurs est atteinte vers 400 secondes. De violents phénomènes de condensation entre cette eau froide arrivant à $50^{\circ} \mathrm{C}$ et la vapeur présente dans le circuit perturbent le comportement de tout le circuit primaire. De brusques variations de pression engendrent des oscillations de débit à travers le cœur, dont le refroidissement reste satisfaisant. Vers 700 secondes, la chute de pression

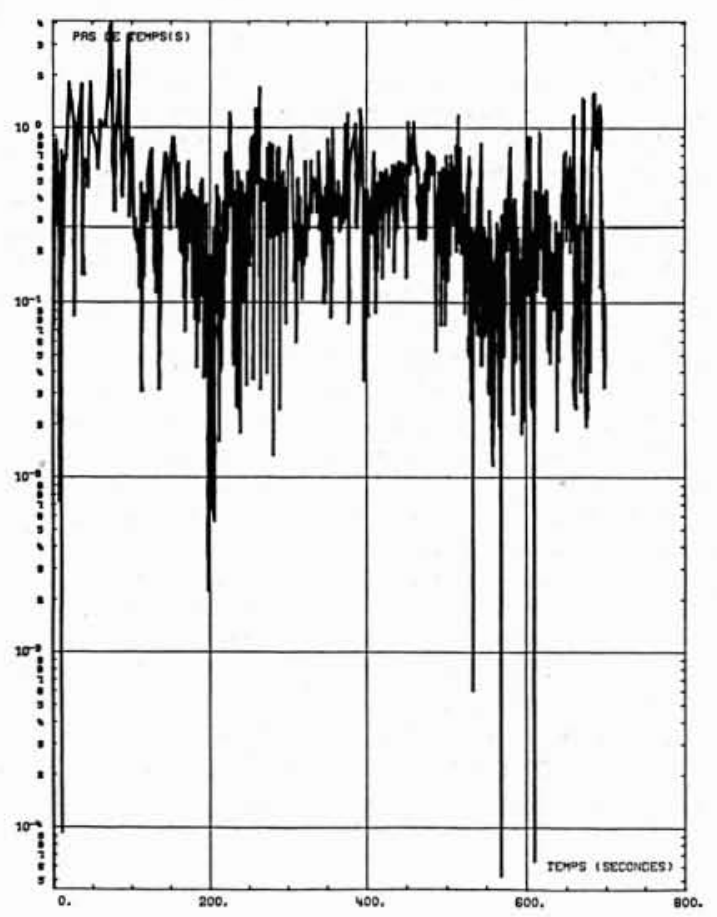

Figure 12 - Evolution du pas de temps en fonction du temps (pas de temps moyen $=0,27$ seconde). est terminée, la pression primaire se stabilise à 18 bars. Les débits injectés (accumulateurs + injections haute pression) compensent la fuite de fluide à la brèche. Rien ne pouvant changer cette situation, l'accident est considéré terminé à 700 secondes.

Le tableau de la figure 4 résume les principaux événements du calcul.

La figure 5 montre l'évolution de la température de gaine au cours du transitoire. On constate que sa valeur maximale $\left(470^{\circ} \mathrm{C}\right.$ à $\left.196 \mathrm{~s}\right)$ est très en dessous du seuil critique. L'intégrité du cœur n'est donc pas atteinte dans ce calcul.

\subsection{Post-traitement des résultats}

L'exploitation des résultats de calcul est faite d'une part à l'aide de tracés de courbes classiques, d'autre part à l'aide de schémas du circuit générés par ordinateur. Les figures 6 à $1 /$ sont des reproductions en noir et blanc de tels schémas. Rassemblant plusieurs milliers d'informations, ils permettent une visualisation synthétique des phénomènes simulés. Les exemples proposés ici concernent les évolutions du taux de vide au cours du transitoire décrit cidessus.

\subsection{Performances}

La simulation de ces 700 secondes de transitoire a nécessité environ 4 heures de temps calcul sur le calculateur CRAY.I.

Le nombre de pas en temps a été de 2583.

La figure 12 donne la valeur de ces pas en temps tout au long du transitoire.

$\mathrm{Ce}$ calcul avait pour objectif la mise au point du code et la démonstration de sa fiabilité. Le prochain objectif sera la diminution du temps de calcul, à la fois par la suppression de la plupart des chutes de pas de temps et par la diminution du temps nécessaire à la résolution d'un pas de temps (environ 5 secondes actuellement).

\section{Références bibliographiques}

[1] Le modèle de base de CATHARE : qualification physique des performances, par J.C. Rousseau (DEDR/DRE/STT). 124 Comité technique de la Société hydrotechnique de France. (Paris, 16 et 17 novembre 1983), La Houille blanche, n’ 3/4, 1984.

[2] Modélisation des volumes dans CATHARE, par J.M. Miraucourt (EDF/SEPTEN). 124 Comité technique de la Société hydrotechnique de France. (Paris, 16 et 17 novembre 1983), La Houille blanche, $\mathrm{n}^{\circ} 3 / 4,1984$. 


\section{Discussion}

Président : M. J. Pelcé

M. MONDIN. - Vous avez déclaré que l'utilisateur devait être "libre, maître et responsable". Qui est l'utilisateur : est-ce le projeteur, l'exploitant, l'organisme de sûreté ? Et comment peut-il exercer sa maîtrise?

M. Miraucourt. - Dans la généalogie même de ce code on retrouve à la fois le projeteur, l'exploitant et l'autorité de sûreté. C'est un code qui est appelé à être utilisé par les trois organismes.

"Libre et maître " c'est pour limiter le nombre de contraintes imposées à l'utilisateur pour la représentation du circuit.

"Responsable ", l'utilisateur doit connaître les hypothèses qui sont faites au niveau des modèles des différents modules de manière à ne pas perdre de vue ce qu'il fait lorsqu'il choisit tel ou tel module pour représenter un composant de circuit.

Le Président. - II doit donc être également compétent ?

M. Miraucourt. - Ce qui ne pose pas de problème pour les utilisateurs de ces trois organismes.

M. FELL - La déformation de la gaine est très sensible à la température, et par conséquent requiert généralement une analyse par sous-canaux. Pouvez-vous préciser comment vous envisagez ce problème dans un modèle global tel que celui de CATHARE ?

M. Miraucourt. - En effet, une représentation détaillée du cceur, par une analyse par sous-canaux par exemple, permet une simulation de son comportement beaucoup plus précise que celle que l'on a actuellement.

Si notre représentation actuelle est suffisante pour les petites brèches, elle est sans doute trop grossière pour les grosses brèches.

Nous sommes conscients de cette insuffisance.

Le Président. - Je vais me permettre de faire un commentaire. On voit là tout l'effort accompli pour obtenir des codes de calcul performants aussi physiques que possible compte tenu des problèmes de transposition dont parlait $M$. REOCREUX. En effet, on compare les codes à des expériences, le réacteur lui-même n'ayant pas eu d'expériences de ce genre, c'est évident.

Cette exigence en matière de représentation physique poussée autant que faire se peut est attachée également aux types, aux catégories de problèmes de sûreté que l'on veut examiner sur ces réacteurs.

Tout à l'heure, M. REOCReux a montré les aspects liés à la notion d'enveloppe, c'est-à-dire ce qui concerne essentiellement la conception du réacteur, savoir si l'on a, en particulier, bien prévu les bonnes dispositions de sauvegarde pour, à coup sûr, ne pas avoir de problèmes sur le combustible en cas d'accident grave sur le réacteur.

Mais la notion d'approche réaliste physique est associée à la façon de conduire le réacteur et à la connaissance de l'opérateur; si, en cours d'exploitation, il devait survenir un accident grave, comment sera-t-il en mesure d'avoir la bonne compréhension des phénomènes qui se passent, et, s'il a les moyens qu'il faut pour réagir, comment pouvoir les utiliser à moins mauvais escient que possible pour résoudre la situation?

Il $y$ a donc une sorte de course entre un certain nombre d'exigences côté objectif de sûreté, dans la connaissance nécessaire et la meilleure possible des phénomènes tels qu'ils sont susceptibles d'intervenir sur le réacteur; et, corrélativement, il y a une pression sur les gens qui élaborent les outils de calcul pour obtenir la meilleure représentation possible.

Tant qu'on ne sera pas venu à bout de la complexité des phénomènes en jeu, on n'aura pas une version ultra-définitive complète, on le sait bien. Mais, en tout cas, pour le moment, ce double souci pousse à l'effort qui est fait.

Voilà le commentaire que je voulais faire, vu du côté de la sûreté des réacteurs.

M. NIGON. - Fait remarquer que les diapositives présentées sont des photos d'écran cathodique dont les images ont été réalisées directement à partir de résultats de calculs sur ordinateur, ce qui représente un travail considérable.

\section{Le Président. - Le suspense continue...}

Nous avons eu ce matin une série d'exposés fort intéressants. Tout d'abod, M. SÉméria a cadré les problèmes. Ensuite nous avons eu des présentations qui abordaient les problèmes en régime diphasique.

J'ai fait un commentaire général sur les pressions qui poussent à l'effort mené dans ce domaine. Je crois qu'il faut bien souligner - et M. Mondin l'a bien fait remarquer - l'étroite liaison qu'il y a entre l'effort théorique qu'il convient de mener, et celui destiné à obtenir un outil d'évaluation pour des applications concrètes. Nous sommes conscients aussi que cet effort de volonté de mise en cuvre de ces outils a une grande influence sur les solutions qu'auront à trouver ceux qui sont plus portés vers le théorique.

Jajouterai un dernier point pour terminer. Je ne vais peut-être pas être très modeste, je m'en excuse, mais je crois que les orateurs le sont trop. Je dirai que l'effort qui est accompli - et non pas seulement par les orateurs de cette matinée, car d'autres compléments vont suivre dans les autres séances en ce qui concerne les codes d'accidents de réacteurs PWR - l'effort mené en France est considéré par la Communauté internationale comme un effort de haut niveau et de grande qualité, et cela devait être dit.

Les Américains font des efforts extraordinaires avec des moyens énormes, il faut le dire aussi, mais la ligne suivie avec CATHARE en France nous fait penser que cet outil, quand il sera au point, sera d'un niveau certainement équivalent aux Codes TRAC ou RELAP de la seconde génération qui sont d'origine américaine. Je crois qu'on peut se risquer à la dire.

Je remercie tous les orateurs et je lève la séance. 